\title{
Presencia docente en ambientes de aprendizaje mediados por Tecnologías de la Información y la Comunicación
}

\section{Teaching presence in learning environments with Information and Communication Technologies}

\author{
John Alexander Rojas-Montero ${ }^{1}$ \\ Sindy Paola Díaz-Better ${ }^{2}$ \\ Universidad Pedagógica Nacional, Colombia
}

Recibido: 05-01-2018

Aceptado: 06-06-2018

\section{Cita Recomendada}

Rojas-Montero, J. \& Díaz-Better, S. (2018) Presencia Docente en Ambientes de Aprendizaje Mediados por Tecnologías de la Información y la Comunicación. Hamut'ay, 5 (1), 53-65. http://dx.doi.org/10.21503/hamu.v5i1.1520

\section{RESUMEN}

Este artículo tuvo el objetivo de presentar los aspectos relacionados con la presencia docente, en una propuesta pedagógica donde se integran objetos de aprendizaje en un ambiente en línea dirigidos al trabajo colaborativo y la transacción educativa. Con fines descriptivos el problema se abordó desde un enfoque cualitativo, para lo cual se diseñó un estudio instrumental de casos en el que participaron estudiantes de estratos 1 y 2 de educación básica primaria de un departamento colombiano. En el estudio de casos se llevaron a cabo las fases preactiva (planeación y diseńo), interactiva (implementación) y postactiva (evaluación) de la propuesta, cuyo interés se centró en la reflexión sobre las tareas, roles y funciones que los docentes deben afianzar antes, durante y al finalizar el proceso para potenciar la transacción educativa y los aprendizajes colaborativos mediados por las Tecnologías de la Información y la Comunicación (TIC). En la dimensión descriptiva del estudio, se encontraron como resultados cualitativos, que los docentes, antes del trabajo con los estudiantes, deben establecer de forma clara los propósitos de aprendizaje, las principales rutas de acción y las situaciones orientadas hacia la motivación para un aprendizaje individual y colaborativo. Durante el tiempo de interacción deben estar atentos al progreso particular y general, a incentivar la participación y a emplear estrategias que posibiliten la construcción de aprendizajes. Al finalizar, los estudiantes reflexionaron sobre si los recursos empleados y las actividades propuestas le dieron sentido a lo aprendido y aplicado por ellos; asimismo si las experiencias en colaboración con otros, promovieron la investigación y la producción continua de conocimientos, interacciones y expresiones.

Palabras Clave: Ambientes de aprendizaje, aprendizaje colaborativo, presencia docente, TIC.

\footnotetext{
1 Docente de la Universidad Pedagógica Nacional (UPN). Magíster en Tecnologías de la Información aplicadas a la educación, UPN. Cursando estudios en el Doctorado Interinstitucional en Educación (DIE) - Sede UPN, Colombia, en el énfasis de Lenguaje y Educación. Director del Grupo de Investigación KENTA-UPN. jarojas@pedagogica.edu.co - jrojas.cursos@gmail.com 2 Docente vinculada a la Secretaría de Educación Distrital (Bogotá). Candidata a Doctora en Educación, Doctorado Interinstitucional en Educación (DIE) - Sede UPN, Colombia. Magíster en Tecnologías de la Información aplicadas a la educación, UPN. Grupo de Investigación KENTA-UPN. spdiaz@educacionbogota.edu.co.
} 


\begin{abstract}
The objective of this article is to present the aspects related to teaching presence, in a pedagogical proposal where learning objects are integrated in an online environment conducive to collaborative work and educational transaction. For descriptive purposes, the problem was addressed from a qualitative approach, for which an instrumental case study was designed, in which students from strata 1 and 2 of basic primary education of a Colombian department participated. In the case study, the pre-active (planning and design), interactive (implementation) and post-active (evaluation) phases of the proposal were carried out, whose interest was focused on the reflection on the tasks, roles and functions that teachers must strengthen before, during and at the end of the process to enhance the educational transaction and collaborative learning mediated by Information and Communication Technologies (ICTs). In the descriptive dimension of the study, it was found as qualitative results, that teachers, before working with the students, must clearly establish the purposes of learning, the main routes of action and the situations oriented towards the motivation for an individual and collaborative learning. During the interaction time, teachers must be alert to the particular and general progress, to encourage participation and to use strategies that make it possible to build learning. At the end, the students reflected on whether the resources used and the proposed activities gave meaning to what was learned and applied by themselves; additionally, if the experiences in collaboration with others, promoted research and the continuous production of knowledge, interactions and expressions.
\end{abstract}

Keywords: Learning environments, collaborative learning, teaching presence, ICT.

\section{INTRODUCCIÓN}

Es usual encontrar en documentos que han sido orientadores de las políticas públicas como Aprender a Ser, La educación del futuro (Faure et al, 1973), Educación para todos y Marco de acción para satisfacer las necesidades básicas del Aprendizaje (WCEFA, 1990); Educación y Conocimiento: Eje de la Transformación Productiva con Equidad (CEPAL-UNESCO, 1996), perspectivas donde se le confiere a la educación un carácter de eje del desarrollo, motor de la sociedad e instrumento de progreso. Esto refleja, como lo señala Álvarez (2013), una lógica económica enfocada hacia los resultados, que entra en tensiones con la educación interesada en procesos permanentes, aleatorios, complejos y contingentes, que han ido mutando gracias, entre otros aspectos, a las dinámicas que las TIC han ido estableciendo, por lo que el aprendizaje actual no remite únicamente a locaciones y tiempos específicos, sino que se puede llevar a cabo de manera constante, lo que requiere tener en cuenta otras disposiciones para aprender y para enseñar.
Estas tensiones evidencian que, "mientras que la enseñanza y la educación han sido, por largo tiempo, consideradas como una fuerza económica, por el contrario, en la sociedad del conocimiento el propio aprendizaje es ahora considerado como potencia productora de valor agregado" (Simons \& Masschelein, 2013, p. 95), sociedad donde se puede crear, acceder, utilizar y compartir información y conocimiento, con el remoto deseo de mejorar la calidad de vida.

Dentro de este panorama, las TIC son aliadas del proceso educativo ya que permiten complementar vivencias formativas a través de nuevos escenarios, recursos y actividades. Aunque no pretenden desplazar o volver obsoleta la figura del docente, es necesario comprender que traen consigo un andamiaje que requiere un compromiso real de quien interactúe con ellas para que no sean vistas solo como artilugios tecnológicos, sino como escenarios que pueden potenciar la construcción colectiva del conocimiento a través de un aprendizaje colaborativo, tanto en el caso de los estudiantes, como en el de los docentes. 
Con estos elementos en juego, se plantea el proyecto de investigación "Promoción del trabajo colaborativo a través de la integración de objetos de aprendizaje en ambientes virtuales: estudio colectivo de casos", llevado a cabo por el Grupo de Investigación KENTA adscrito a la Universidad Pedagógica Nacional (Colombia). El objetivo general del proyecto fue caracterizar desde una experiencia investigativa concreta ${ }^{3}$, algunas de las tareas y roles que se requieren asumir desde la presencia docente en el proceso de inclusión de las Tecnologías de la Información y la Comunicación (TIC) en ambientes de aprendizaje con enfoque colaborativo.

Como objetivos específicos se plantearon, por un lado, diseñar una propuesta pedagógica para la promoción del trabajo colaborativo y la transacción educativa mediada por las TIC; por otro, implementar la propuesta pedagógica con estudiantes de educación básica primaria; y, por último, evaluar la propuesta a partir de los resultados obtenidos en el ambiente sobre el trabajo colaborativo, la transacción educativa y la presencia docente.

En la propuesta pedagógica mediada por un ambiente de aprendizaje en línea, confluyeron las presencias que proponen Garrison \& Anderson (2005) al integrar aspectos desde lo cognitivo, lo social y lo docente, interesando particularmente esta última presencia, ya que, al incorporar otros escenarios de aprendizaje mediados por TIC, se requiere una resignificación de los roles tanto de estudiantes como de docentes.

Roles de la presencia docente en ambientes de aprendizaje

Las TIC en el ámbito educativo han generado un cambio sustancial en la presencia docente, sea en la modalidad presencial, virtual o a distancia, que ha repercutido en los roles docentes. Este cambio ha obligado a los docentes a desarrollar ciertas

\footnotetext{
3 El proyecto de Investigación "Promoción del trabajo colaborativo a través de la integración de objetos de aprendizaje en ambientes virtuales: estudio colectivo de casos" (DTE-149-09), retoma algunas experiencias investigativas de docentes pertenecientes al Semillero de Investigación KENTA de la UPN en el período de 2008-2009, siendo una de ellas la desarrollada por la docente Elizabeth Ayala, de la cual se tuvieron en cuenta algunos elementos para el desarrollo de este artículo.
}

competencias digitales, movilizando sus conocimientos, habilidades y actitudes para utilizar las TIC en su práctica docente (Escorcia-Oyola \& Jaimes, 2015; Fainholc et al, 2015; Bustos \& Román, 2016; Luján, 2016; Viñals \& Cuenca, 2016). Al respecto, Camacho (2014) luego de hacer una revisión bibliográfica alrededor de las competencias docentes en el uso de las TIC, concluye que, con mínimas variaciones, existe un consenso en identificar roles asociados a lo pedagógico, a la gestión, y a componentes sociales y técnicos, pero en ocasiones es difícil identificar dichos roles desde la experiencia práctica de la educación en línea. Según Luján (2016), son los docentes quienes deben estar más comprometidos, por el éxito de una innovación pedagógica mediada por tecnologías porque dependerá en gran medida del nivel de conocimiento que estos tengan al respecto, ya que:

"el docente como guía, facilitador o tutor no solo debe de poseer competencias pedagógicas inherentes a su labor, sino debe de estar a la par del avance de las TIC, por lo que se hace imprescindible que desarrolle competencias tecnológicas, digitales o informacionales en su quehacer cotidiano dentro y fuera del aula" (Luján, 2016, p. 24).

Este cambio de rol frente a las mediaciones tecnológicas precisa que el docente haga uso de ellas y sea capaz de identificar, planear, estimar y proponer otros modos que motiven el aprendizaje y a la construcción colectiva y colaborativa de experiencias de aprendizaje entre pares. Ruíz \& Aguirre (2013) señalan que, ante la incursión de las TIC, los docentes se han posicionado como mediadores del aprendizaje, al facilitar recursos, orientar prácticas pedagógicas y diseñar ambientes, cediendo su papel protagónico de antaño, al dar el control al estudiante en su propio aprendizaje; ya que tienen la oportunidad de ampliar su conocimiento utilizando las TIC como herramientas de apoyo (Farroñay \& Ancaya, 2016).

En los entornos de aprendizaje mediados por las TIC, el papel del docente no recae únicamente en ser un facilitador de la interacción con unos contenidos y actividades. Para realizar un papel idóneo en estos entornos, es casi una exigencia 
ética haber sido (y continuar siendo) un aprendiz en estos ámbitos y escenarios lo cual cobra importancia al tener en cuenta que, como señala Larrosa (2003), "nadie debe aceptar dogmáticamente la experiencia de otro y nadie puede imponer autoritariamente la propia experiencia a otro" (p.4).

Estas posturas traen a la discusión otro hecho relevante relacionado con la identificación de funciones y tareas adicionales que se requieren desarrollar por parte del docente, ya que, como señala Gutiérrez (2016), aunque la función principal del docente es contribuir al desarrollo de las competencias de sus estudiantes, no es la única.

Otros autores recalcan el rol del docente como moderador, encargado de fomentar el aprendizaje en un diálogo en línea o en un área de discusión en red (Collison, Elbaum, \& Haavind, 2000; Olaizola \& Peralta, 2017; Rodríguez \& López, 2017), es decir, cumplir el papel de mediar entre los miembros del grupo y facilitar todos los elementos a su alcance para que logren una situación real de aprendizaje, desarrollando previamente la habilidad para analizar lo que está pasando y enfocar el trabajo hacia los caminos más apropiados para llegar a un progreso de consenso. Por su parte Abdous (2011) hace referencia a las nuevas tareas y competencias docentes a partir de una propuesta que integra tres fases no lineales, con respecto a lo que se debe hacer antes (tareas de planificación y diseño), durante (roles relacionados con la facilitación, la interacción y la retroalimentación), y después (roles que permitan la reflexión de lo aprendido) de la intervención con ambientes de aprendizaje en línea. En la Figura 1 se presentan algunos conceptos asociados con el rol docente como moderador.

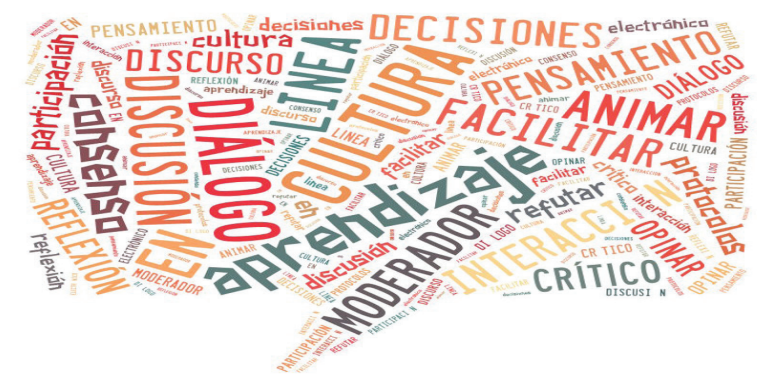

Figura 1 Conceptos asociados al rol de Moderador en ambientes mediados por TIC

Fuente: Elaboración propia, creado en Tagxedo (2016).
Por su parte, Viñals \& Cuenca (2016) señalan que, si bien la información es abundante en la red, dentro de las tareas que ahora deben cumplir los docentes que hagan uso de las TIC se encuentran guiar a los estudiantes en procesos de búsqueda, análisis y selección de información relevante, interpretación de datos, síntesis de contenidos y elección de estrategias adecuadas para difundirlos.

Presencia docente en ambientes de aprendizaje mediados por tecnologías

De acuerdo con los objetivos planteados previamente, interesa ampliar el concepto de presencia docente. Para Garrison \& Anderson (2005), la presencia docente hace referencia a todas aquellas actividades y acciones que facilitan y orientan los procesos de carácter cognitivo y social en un ambiente de aprendizaje en línea, con el objetivo de propiciar aprendizajes significativos, para lo cual se deben tener en cuenta las necesidades y capacidades de los estudiantes.

La presencia docente en ambientes mediados por TIC requiere que se pueda entablar una relación empática con los estudiantes en forma temprana; que exista una claridad en el proceso de aprendizaje y en la evaluación; y que se establezcan las estrategias oportunas para la participación y el debate; ya que existe una gran posibilidad de deserción en el proceso. En otras palabras, recae sobre la figura docente, tareas y funciones que debe desempeñar idóneamente en pro del éxito de la experiencia de enseñanza y de aprendizaje. Llorente (2006), propone que un docente en línea debe desempeñar tareas relacionadas con aspectos: (1) Tecnológicos, como la gestión de la información en las plataformas que se empleen. (2) Académicos, relacionados con el diseńo de actividades de aprendizaje, con propósitos definidos; realimentación y evaluación de estas. (3) Sociales: tendientes a animar la participación, dinamizar el trabajo en equipo y conducir las intervenciones. (4) De orientación, para aclarar, motivar, guiar e informar sobre el proceso de los estudiantes. (5) Organizativos, para planear, coordinar el ambiente de aprendizaje y las actividades que allí se lleven a cabo. 
Mauri \& Onrubia $^{4}$ (2008) plantean un recorrido por las competencias y perfil del docente en estos escenarios de formación, centrándose en la dimensión tecnológica y en la construcción de conocimiento. Desde la dimensión tecnológica sugieren que el docente en línea debe estar ampliamente familiarizado con la tecnología desde un componente teórico y práctico, que le permita valorar tanto el papel de las TIC en la educación y enseñanza, como las implicaciones y consecuencias de estas. Se requiere además que el profesor sea eficaz en la búsqueda y selección de recursos ya existentes, pero que también sea competente para diseñar materiales que involucren las TIC.

La presencia docente en la propuesta pedagógica desarrollada para el proyecto "Promoción del trabajo colaborativo a través de la integración de objetos de aprendizaje en ambientes virtuales" (KENTA, 2009), buscó articular los roles y funciones para mantener la motivación de los estudiantes, analizar sus aportes y promover la reflexión con el propósito de orientar, enriquecer y realimentar el proceso de enseñanza - aprendizaje al realizarse trabajo colaborativo diseñando una serie de hechos desencadenantes. La Figura 2 presenta en forma general los roles del docente en la propuesta desarrollada.

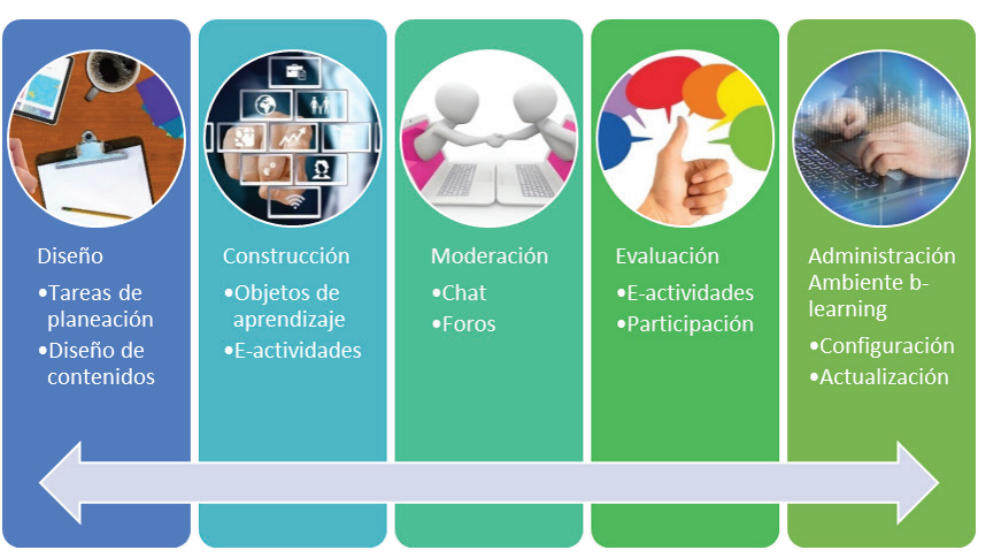

Figura 2 Presencia Docente en la propuesta pedagógica Fuente: Elaboración propia. Imágenes de uso gratuito (Pixabay)
Tecnologías de la Información y la Comunicación (TIC)

El concepto de Tecnologías de la información y Comunicación admite diversas miradas para su comprensión. Una de ellas se acerca desde lo instrumental, al referirse como una disciplina que busca resolver diversas necesidades; que usuarios en contextos específicos puedan tener, para lo que se hace uso de dispositivos o servicios relacionados con la tecnología (ACM, 2005). Otra mirada tiene que ver con los procesos que permiten llevar a cabo, entre los que se encuentran los relacionados con la adquisición, la producción, el almacenamiento, el tratamiento y la transmisión de información, (digital principalmente) desde diversos formatos (Duncombe \& Heeks, 1999; Grande, Cañón \& Cantón, 2016), procesos que según Jiménez (2009), están destinados a optimizar la comunicación humana. Finalmente, desde las posibilidades que las TIC ofrecen a las comunidades que hacen uso de ellas en distintos ámbitos, ya que han transformado la forma en que los seres humanos establecen relaciones sociales, se entretienen, adquieren bienes de consumo o servicios, o aprenden. (González, 2008; Gasca, Martínez \& Tautiva, 2016, Vinueza \& Simbaña, 2017). Las TIC han permitido la creación de otros escenarios de trabajo, de aprendizaje, de comunicación, de interpretación y creación subjetiva; por lo que ahora también son reconocidas como escenarios que permiten a los sujetos conocer, interactuar y expresar su sentir a través de diversos medios y formatos; lo que hace indicar, que las TIC, como refiere Fernández (2016), han dado lugar a la transformación de la Sociedad Industrial para pasar a una sociedad de la información en la que se generan diversas estéticas (Scolari, 2008).

En el panorama educativo, las TIC hacen converger a estudiantes, docentes y recursos en espacios diseñados según las necesidades; incidiendo en los modos de ser, de comprender, de compartir y de producir conocimiento; "ya que ofrecen una gama de posibilidades interactivas para el aprendizaje y el establecimiento de la comunicación mediante el desarrollo de actividades colaborativas" (Montenegro, 2016, p. 69); sin embargo, es importante señalar que no se trata de repetir a través de plataformas tecnológicas lo que ocurre 
en ambientes presenciales.

Entre las distintas modalidades de incorporación de las TIC en el campo educativo encontramos las híbridas (blended learning, o b-learning), entendidas como un modelo de aprendizaje donde se combinan actividades de enseñanza orientadas tanto de forma presencial como en línea, que permiten estructurar escenarios educativos más ricos y variados a través de estas tecnologías (Contreras, Alpiste \& Eguia, 2006; Najmanovich, 2009; Sein-Echaluce, Fidalgo \& García, 2015, Cerón, Gómez \& Abrego, 2015).

En el b-learning, tal como lo señala Sánchez (2015), el docente profundiza en estrategias que faciliten un aprendizaje autónomo de los estudiantes, tanto desde lo cognitivo como lo actitudinal. Al respecto, en el proyecto "Promoción del trabajo colaborativo a través de la integración de objetos de aprendizaje en ambientes virtuales: estudio colectivo de casos", se diseñó en un ambiente b-learning a partir de las presencias planteadas por Garrison \& Anderson (2005), que corresponden a los elementos básicos que deben considerarse si se desea planificar y estructurar una experiencia mediada por TIC, y que son explicadas en la Tabla 1.

Tabla 1. Presencia en ambientes de aprendizaje en línea según Garrison \& Anderson (2005)

\begin{tabular}{ll}
\hline \multicolumn{1}{c}{ Presencia } & \multicolumn{1}{c}{ Descripción } \\
\hline Presencia cognitiva & $\begin{array}{l}\text { Hace referencia a los resultados educati- } \\
\text { vos que se pretenden alcanzar mediante } \\
\text { procesos de reflexión continua }\end{array}$ \\
\hline Presencia social & $\begin{array}{l}\text { Se evidencia desde la interacción entre los } \\
\text { miembros de una comunidad a través de } \\
\text { diversos medios y canales de comunicación }\end{array}$ \\
\hline Presencia docente & $\begin{array}{l}\text { Involucra distintas tareas de planeación y } \\
\text { orientación que realiza quien esté a cargo } \\
\text { de un ambiente de aprendizaje. }\end{array}$ \\
\hline
\end{tabular}

Fuente: Elaboración a partir de Garrison y Anderson (2005)

En este contexto aparece el aprendizaje colaborativo, como interdependencia de los miembros de un grupo que comparten ideas y experiencias únicas; que trabajan sobre propósitos comunes, confrontan ideas, crean significado juntos, se enriquecen y crecen mutuamente, lo que hace que sus contribuciones con frecuencia no se puedan separar claramente al final del proceso (Silva, 2007; Freire,
Gómez \& García, 2016). En ambientes mediados por TIC, lo colaborativo se traduce a una construcción de comunidad, donde se requiere el establecimiento de metas comunes que no se puedan lograr en forma individual (Driscoll \& Vergara, 1997 citado en García-Gasco, 2003; Correa, Sierra \& Alzate, 2015); la responsabilidad compartida para el cumplimiento de esas metas (Cabero \& Llorente, 2007; Gutiérrez-Santiuste \& Gallego-Arrufat, 2017) y la construcción a partir de los consensos (Graham \& Misanchuk, 2004; Vega, 2017). Estas características requieren de mayor compromiso de los estudiantes y docente para el cumplimiento de acuerdos que posibiliten una interacción adecuada y productiva al interior de los escenarios de aprendizaje mediados por las TIC.

A partir de estas consideraciones en el estudio se diseñó una propuesta pedagógica que promovía el trabajo colaborativo a partir de la integración de Objetos de Aprendizaje en un Ambiente Virtual de Aprendizaje (AVA) y el planteamiento de actividades para la construcción de conocimiento y el mejoramiento de la transacción educativa.

El tema abordado fue el de sistemas, como parte de la conceptualización que se desarrolla en el área de Tecnología e Informática sobre sistemas tecnológicos. Para llegar al concepto de sistema y sus características, las temáticas se distribuyeron en tres módulos, partiendo de una parte del análisis de un sistema natural (Sistema solar); en el segundo módulo interesó un sistema artificial (sistema básico de circuitos); para finalizar con uno que integra los conocimientos adquiridos en los módulos 1 y 2 (Ayala, 2009). En cada módulo se integró un Objeto de Aprendizaje, partiendo de una intencionalidad, una situación particular que interrogó sobre los temas, una invitación al aprendizaje y una e-actividad (Cabero \& Román, 2008; Silva, 2017). A partir de una situación inicial se activan conocimientos previos, se aborda una lectura sobre el tema, se desarrolla una actividad y se realiza una pequeña evaluación (Ver Figura 3).

Los estudiantes contaron permanentemente con acceso a la sala de Chat como recurso de apoyo y de encuentro para resolver colaborativamente la actividad propuesta. 


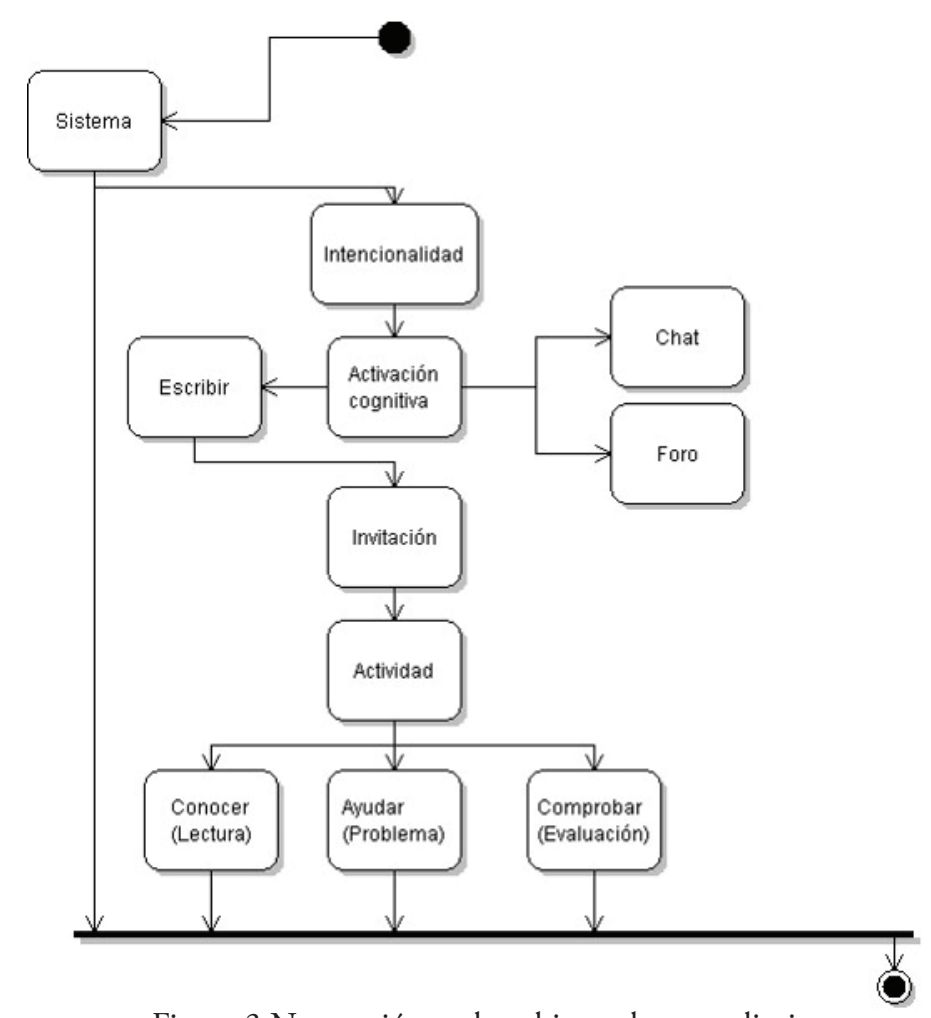

Figura 3 Navegación en los objetos de aprendizaje Fuente. Informe KENTA (2009)

Considerando el proceso de potenciar el aprendizaje colaborativo las e-actividades se orientaron a buscar participación y trabajo en equipo para las percepciones de los otros, admitiendo el valor de sus opiniones y potenciando el intercambio y búsqueda de consenso. La preparación y socialización se realizó a partir de foros.

Las tareas asociadas a la administración del aula b-learning, se enfocaron al manejo de los recursos pertinentes (Chat, foros, ejercicios, material de trabajo); la actualización de contenidos y actividades; el registro de participantes del aula; configuración y la habilitación de módulos de acuerdo con su pertinencia y necesidad dentro del aula.

Como los estudiantes no habían trabajado previamente con escenarios de aprendizaje en línea se realizaron actividades de familiarización con la navegación y el uso de foros y chat. Al ser un ambiente b-learning, se desarrollaron algunas actividades presenciales a partir de los recursos del ambiente. Los objetos de aprendizaje permitieron el reconocimiento de conceptos y el desarrollo de ciertas actividades evaluativas (Ayala, 2009).

En el ambiente de aprendizaje el texto escrito se enriqueció con diversos formatos o morfologías de comunicación, como son la animación, el audio y las simulaciones (Rodríguez, 2007; Arce, Rodríguez \& Husted, 2016), con el fin de generar múltiples interacciones entre recursos audiovisuales y textuales, lo que Henao (2001) justifica al indicar que el usuario de estos entornos trata de construir significados mediante la exploración de esos recursos.

\section{Materiales Y Métodos}

\section{Participantes}

Los participantes fueron estudiantes que pertenecían a una institución pública que fue seleccionada por contar con las condiciones tecnológicas requeridas para el trabajo investigativo. Pertenecientes a un estrato socioeconómico 1 y 2 , y en general con escaso dominio de las TIC debido a que en sus hogares rara vez están presentes. Estos estratos se caracterizan por bajos ingresos, situación de desempleo critica, poca capacidad de responder a sus gastos básicos, así como baja calidad y cantidad de habitaciones en los sitios de vivienda. Fueron 10 los estudiantes que participaron del estudio, siendo el promedio de edad entre los 10 y 12 años, quienes tenían un dominio tecnológico suficiente para participar en el estudio.

\section{Instrumento}

Como instrumentos se utilizaron los foros y chats (Rodríguez \& Vallderiola, 2001; Orellana \& Sánchez, 2006; Álvarez, 2009; Carmona, 2011; Colmenares, 2012) presentes en cada uno de los módulos del ambiente en línea. Estos instrumentos permitieron registrar las participaciones de los estudiantes durante su interacción con los objetos de aprendizaje, con los cuales fue posible describir los siguientes aspectos que fueron de interés durante el desarrollo de la investigación: (1) el ambiente de aprendizaje con sus dimensiones de trabajo colaborativo (negociación de sentidos, distribución de saberes) y transacción educativa (transmisión de conocimiento, producción de conocimiento); (2) la presencia docente con sus dimensiones de planeación, ejecución y evaluación. Los datos recopilados en foros y chat pasaron por 
un proceso de análisis de contenidos, que permitió determinar lo que un docente debía tener en cuenta antes, durante y después del desarrollo de las actividades que propone a sus estudiantes, y así tener elementos para reflexionar acerca de cómo mejorar las condiciones para que sus estudiantes tengan éxito en su proceso de aprendizaje.

\section{Tipo y diseño}

La investigación se llevó bajo un enfoque cualitativo. Siendo importantes las experiencias y percepciones de los participantes se enfocó en el proceso investigativo como en los posibles resultados, empleando la descripción como forma de presentar los resultados (Creswell, 2003). Así, se describe lo ocurrencia al integrar objetos de aprendizaje en un ambiente en línea, con respecto al trabajo colaborativo y a la transacción educativa, utilizando para ello un estudio instrumental de caso llevado a cabo en sus fases preactiva (Fase uno y dos del proyecto), interactiva (Fase 3 del proyecto) y postactiva (Fase 4 del proyecto) (Álvarez $\&$ San Fabián, 2012). Al analizar la incidencia de tres objetos de aprendizaje en la promoción del trabajo colaborativo, cada objeto se constituyó en un caso de estudio independiente, empleando las actividades propuestas para motivar la interacción a través de foros y chat entre los estudiantes participantes para llegar a una solución conjunta de un problema planteado.

\section{Procedimiento}

Se llevaron a cabo cuatro fases (ver Figura 4). En la Fase uno se realizó el análisis del dominio de conocimiento para el que se desarrollaron los objetos de aprendizaje, que permitió la delimitación del proyecto; el establecimiento de los requerimientos de hardware y software; la contextualización del ambiente tecnológico; la definición del perfil del estudiante. En la Fase dos se diseñó la propuesta pedagógica enfocada en la promoción del trabajo colaborativo a partir de la integración de Objetos de Aprendizaje en un Ambiente en línea y la elaboración de e-actividades.

En la Fase tres: se implementó la propuesta con el fin de poner a disposición de estudiantes de grado quinto el ambiente y los objetos de aprendizaje.
En la Fase cuatro se evaluó la propuesta a partir de la reflexión sobre lo ocurrido y los aspectos relacionados con la presencia docente, a partir del análisis de la información recolectada.

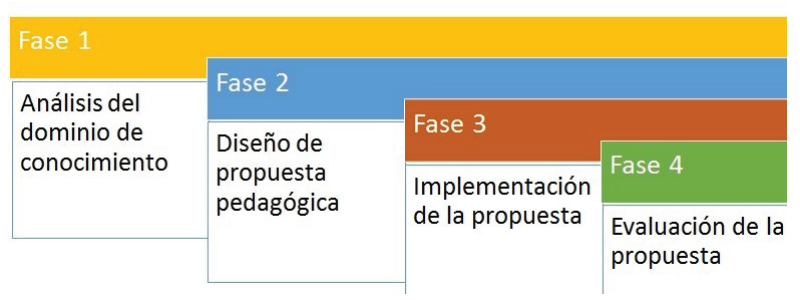

Figura 4. Fases de la investigación

Fuente. Elaboración propia (2016)

Confidencialidad. Por el principio de confidencialidad, se omiten los nombres de los participantes, a quienes se les identificó con la letra $\mathrm{E}$ y un número, de acuerdo con el orden en los registros.

\section{Resultados}

Para el enfoque investigativo cualitativo con fines descriptivos, se presentan los resultados del análisis de contenido de los foros y los chats, como evidencia de lo ocurrido con el ambiente de aprendizaje y la presencia docente.

\section{El ambiente de aprendizaje}

En el Módulo 1 (Tabla 2) se observa que los estudiantes compartieron conocimientos previos y hubo tres aspectos comunes en sus respuestas: las que relacionan el Sol con el planeta Tierra y otros planetas; las respuestas que hicieron referencia a la influencia desde el clima y la formación del día y la noche; y las que hablaron solo de la luna y el planeta Tierra. Las relaciones que se identificaron fueron en su mayoría binarias y no involucraron en sus respuestas a todos los elementos del Sistema Solar. Las respuestas se presentaron como bloques individuales. Por otro lado, los estudiantes no hicieron comentarios sobre las respuestas de sus compańeros, solo dieron la respuesta a la pregunta. Solo un estudiante manifestó explícitamente estar de acuerdo con la participación de sus compañeros sin dar ningún aporte o explicación adicional. 
Tabla 2 Respuestas Foro Módulo 1

\begin{tabular}{lllllllll}
\hline $\begin{array}{l}\text { Pregunta } \\
\text { motivadora }\end{array}$ & \multicolumn{6}{c}{$\begin{array}{l}\text { Qué relaciones identificas entre los componentes del sistema solar? } \\
\text { (Claves: S: sol - T: Planeta Tierra - L: Luna - P: otros Planetas - E: Estudiante) }\end{array}$} \\
\hline $\begin{array}{llllllll}\text { Relaciones } \\
\text { identificadas }\end{array}$ & $\begin{array}{l}\text { S-T/ } \\
\text { S-P }\end{array}$ & $\begin{array}{l}\text { L, S } \\
\text { T }\end{array}$ & $\begin{array}{l}\text { Climal } \\
\text { Día-No- } \\
\text { che }\end{array}$ & $\begin{array}{l}\text { Influencia } \\
\text { luna en el } \\
\text { mar }\end{array}$ & $\begin{array}{l}\text { Relacionaron } \\
\text { otros compo- } \\
\text { nentes }\end{array}$ & $\begin{array}{l}\text { Comparte } \\
\text { respuestas }\end{array}$ & $\begin{array}{l}\text { No res- } \\
\text { ponde }\end{array}$ \\
\hline Participante & E1 & E1 & E1 & E1 & E6 & E2 & E8 & E9 \\
& & E2 & E4 & E3 & & & & E10 \\
& E4 & E5 & E4 & & & & \\
& E5 & E6 & E6 & & & & \\
& E6 & & E7 & & & & \\
\hline
\end{tabular}

Fuente: Elaboración propia a partir de Anexo C, Ayala (2009)

En el módulo 2 la participación fue más homogénea comparado con el módulo 1. Algunos estudiantes replicaron la información dejada en la situación problema y solo dos estudiantes ampliaron su respuesta en forma más acertada (Tabla 3).

Tabla 3. Respuestas Foro Módulo 2

\begin{tabular}{lllll}
\hline $\begin{array}{l}\text { Pregunta } \\
\text { motivadora }\end{array}$ & \multicolumn{2}{c}{$\begin{array}{c}\text { ¿Cómo puedes ayudar a través de un sistema de } \\
\text { circuitos a que se escuche el timbre en el lugar } \\
\text { más alejado de la casa? }\end{array}$} \\
\hline Respuestas & $\begin{array}{l}\text { Menciona } \\
\text { algunos } \\
\text { compo- } \\
\text { nentes }\end{array}$ & $\begin{array}{l}\text { Repite ele- } \\
\text { mentos de } \\
\text { la pregunta }\end{array}$ & $\begin{array}{l}\text { Da una } \\
\text { respuesta } \\
\text { completa } \\
\text { Sistema }\end{array}$ & $\begin{array}{l}\text { No } \\
\text { responde }\end{array}$ \\
& y más & \\
\hline Participante & E1 & E2 & E5 & E5 \\
& E7 & E3 & E6 & E6 \\
& E8 & E4 & & \\
& & E9 & & \\
\hline
\end{tabular}

Fuente: Elaboración propia a partir de Anexo C, Ayala (2009)

En las sesiones de Chat no todos los estudiantes participaron y la intervención docente fue para aclarar las dudas de los estudiantes (Ver Tabla 4).

Tabla 4. Participación en Chat

\begin{tabular}{|c|c|c|c|c|c|}
\hline Sesión & $\begin{array}{l}\text { Partici- } \\
\text { pantes }\end{array}$ & $\begin{array}{l}\text { Dudas } \\
\text { sobre } \\
\text { procedi- } \\
\text { miento/ } \\
\text { problema }\end{array}$ & $\begin{array}{l}\text { Comenta- } \\
\text { rios sobre } \\
\text { respues- } \\
\text { tas } \\
\text { propias }\end{array}$ & $\begin{array}{l}\text { Acep- } \\
\text { tación } \\
\text { pasiva }\end{array}$ & $\begin{array}{l}\text { Aclaración } \\
\text { procedi- } \\
\text { miento }\end{array}$ \\
\hline \multirow[t]{3}{*}{1} & E1 & $X$ & & $X$ & \\
\hline & E2 & $x$ & $X$ & $x$ & $X$ \\
\hline & E6 & & $X$ & & \\
\hline \multirow[t]{2}{*}{2} & E2 & $X$ & & & \\
\hline & $D^{*}$ & & & & $X$ \\
\hline
\end{tabular}

Fuente: Elaboración propia a partir de Anexo C, Ayala (2009)

* Registro docente
La promoción del trabajo colaborativo y la transacción educativa también apareció en las sesiones de trabajo presencial, aunque en ocasiones algunos estudiantes tomaron un papel pasivo, ya que sólo retomaron los aportes textuales de sus compañeros o los aceptaron sin hacer ningún aporte adicional.

\section{Presencia docente}

La presencia docente se evidenció, en primera instancia en el trabajo previo a la validación del ambiente de aprendizaje, en las tareas de administración, diseño y planificación, lo que muestra que en ambientes de aprendizaje mediados por TIC la presencia docente se realiza desde mucho antes de la interacción con los estudiantes y requiere una construcción previa de rutas posibles de aprendizaje, selección de recursos disponibles, construcción de metas de aprendizaje particulares para poder proyectar las actividades. De igual forma cabe señalar que, si bien se realizó un camino previo de implementación que sirvió de ruta principal, tal como ocurre en ambientes presenciales, al momento de la interacción con los estudiantes y el ambiente, se hizo necesario replantear algunos de ellos si el resultado no fue el esperado de acuerdo con las metas propuestas.

Sin embargo, no se evidenció un acompañamiento permanente en las actividades en línea, lo que pudo influir en la baja participación. Los procesos de moderación e interacción con estudiantes a través de ambientes mediados por TIC tienen por fines principales centrar la atención en los puntos más importantes de las temáticas abordadas, 
tratando de generar posturas reflexivas frente a los mismos, más que replicar un conocimiento ya establecido por otros. La presencia docente cobra aquí más visibilidad debido a que puede influir en la continuidad del proceso. No se presentó ningún cierre de las participaciones, retroalimentación de las respuestas en las formas de comunicación utilizadas, aclaraciones sobre las temáticas y sobre las concepciones no veraces de los participantes; la intervención se limitó a recordar algunos aspectos procedimentales y actividades pendientes. A continuación, algunas de estas intervenciones en forma literal (Ayala, 2009):

- "No te olvides que tienes una prueba final para demostrar lo que has aprendido..."

- "Si no estás tan seguro puedes revisar nuevamente el tema"

- "Recuerda que hay otros dos temas que trabajar"

\section{Discusión y Conclusiones}

Si bien se presentaron indicios de una promoción del trabajo colaborativo y la transacción educativa, los resultados no permiten ser concluyentes al respecto, lo que puede estar relacionado con la baja presencia docente en los espacios de interacción diseñados. Como lo señala Abdous (2011), la presencia docente en ambientes de aprendizaje requiere el desarrollo de tareas antes, durante y después del trabajo con los participantes.

Antes, porque es necesario establecer en forma clara los propósitos de aprendizaje, determinar las rutas principales de acción; planear cuidadosamente y en forma creativa las situaciones que motiven un aprendizaje individual y colaborativo; aspectos que recogen ampliamente los aportes de Llorente (2006); Mauri \& Onrubia, (2008) en Coll \& Monereo, (2008) y que se evidenciaron en la experiencia evaluada.

Por otro lado, durante el tiempo que se interactúe con los ambientes de aprendizaje diseñados, el docente debe estar atento al progreso particular y general; motivar la participación; aplicar las estrategias planeadas y desarrollar otras que posibi- liten la construcción de aprendizajes, si las metas comunes no se están alcanzando adecuadamente; para ello, no solo debe tener un amplio conocimiento en los aspectos relacionados con las temáticas específicas que se proponen, sino habilidades comunicativas que le permitan favorecer la participación y centrar el debate entre ellos, ya que las participaciones de los estudiantes pueden fomentar o interrumpir el flujo de la comunicación, tal como propone Díaz (2008). Estos aspectos fueron insuficientes, ya que los estudiantes realizaron aportes individuales al foro, pero no recibieron retroalimentación de estos, pese a que algunas respuestas por parte de los estudiantes requerían de una aclaración.

Lograr una adecuada presencia docente en ambientes de aprendizaje colaborativo mediados por TIC exige apropiarse de los nuevos roles adscritos a ella; teniendo presente que hay distintas etapas de planeación, construcción de recursos, administración e interacción y moderación con quienes participan; pasando de un papel tradicional que le confiere a veces una responsabilidad de transmisión; y aportando desde una visión holística a que se analicen, interpreten y propongan otras miradas, que pueden devenir en una construcción colectiva del conocimiento.

No se trata de privilegiar algunas tareas o momentos sobre otros, pero sí se hace necesario hacer una lectura holística, ya que, como señala Camacho (2014):

“... esta nueva forma de entender el rol de los docentes y sus competencias ha permitido una transformación integral donde una cantidad importante de nuevos enfoques dentro del ambiente educativo están presentes, y que no eran entendidos ni asumidos algunos ańos atrás” (p. 622).

Para ello, el docente en ambientes mediados por TIC debe buscar, gestionar y presentar recursos $\mathrm{y}$ actividades que interroguen sobre el sentido de lo que se aprende y la manera de aplicar lo aprendido en contextos específicos, propiciando así un proceso contextualizado que posibilite la construcción de experiencias en colaboración con otros, donde se promueva la investigación, la creación y la producción intelectual continua. 


\section{Agradecimientos}

Este artículo se deriva del proyecto de investigación "Promoción del trabajo colaborativo a través de la integración de objetos de aprendizaje en ambientes virtuales: estudio colectivo de casos", que fue financiado por el Centro de Investigaciones de la Universidad Pedagógica Nacional (CIUP), y es reconocido con el código DTE-149-09. Expresamos nuestro agradecimiento a la docente Elizabeth Ayala, miembro del Semillero de Investigación KENTA UPN en el periodo 2008-2009 durante su proceso de formación como especialista, y cuyo trabajo se retoma en algunos apartados de este artículo.

\section{REFERENCIAS BIBLIOGRÁFICAS}

Abdous, M. (2011). A Process-Oriented Framework for Acquiring Online Teaching Competencies. Journal of Computing in Higher Education, 23(1), 60-77. https://doi. org/10.1007/s12528-010-9040-5

ACM. (2005). Computing curricula: Information Technology Volume. Recuperado de http://www.acm.org/education/curric_vols/IT_October_2005.pdf

Álvarez, Á. \& San Fabián M. (2012). La elección del estudio de caso en investigación educativa. Gazeta de Antropología, 2012, 28(1). Recuperado de http://www.ugr.es/-pwlac/ G28_14Carmen_Alvarez-JoseLuis_SanFabian.html

Álvarez, C. (2009). Etnografía virtual: exploración de una opción metodológica para la investigación en entornos virtuales de aprendizaje. Revista Q, 3(6). Recuperado de https://revistas.upb.edu.co/index.php/revista_Q/article/ view/7809

Álvarez, A. (2013). La mirada empresarial de la educación. A propósito del informe Compartir. Revista Pedagogía y Saberes, (39), 141-165. http://dx.doi.org/10.17227/0121249 4.39pys 140.165

Arce P., Rodríguez G. \& Husted R. (2016). La importancia del desarrollo de instrumentos para la evaluación de la comunicación interactiva y el lenguaje multimedia en entornos virtuales de enseñanza-aprendizaje (EVE-A). En: Actas del I Congreso Internacional Comunicación y Pensamiento. Comunicracia y desarrollo social (2016), 12551271. Egregius. Recuperado de https://idus.us.es/xmlui/ handle/11441/51486

Ayala, E. (2009). Trabajo colaborativo y objetos de aprendizaje en un ambiente virtual para la enseñanza de los sistemas. Bogotá: Trabajo de Grado Especialización TIAE. Universidad Pedagógica Nacional.
Bustos, A. \& Román, M. (2016). La importancia de evaluar la incorporación y el uso de las TIC en educación. Revista Iberoamericana de evaluación educativa, 4(2). Recuperado de https://revistas.uam.es/index.php/riee/article/view/4452

Cabero, J. \& Llorente, M. (2007). Propuestas de colaboración en educación a distancia y tecnologías para el aprendizaje. Edutec. Revista electrónica de tecnología educativa, (23). Recuperado de http://www.edutec.es/revista/index. php/edutec-e/article/view/492/226

Cabero, J. \& Román, P. (2008). E- actividades, Un referente básico para la formación en Internet. Bogotá: Magisterio.

Camacho, L. (2014). Nuevos roles de los docentes en la educación superior: hacia un nuevo perfil y modelo de competencias con integración de las TIC. Ciencia y Sociedad, 39(4), 601-640. https://doi.org/10.22206/cys.2014.v39i4. pp601-640

Carmona J. (2011). Tensiones de la etnografía virtual: teoría, metodología y ética en el estudio de la comunicación mediada por computador. F@ ro: revista teórica del Departamento de Ciencias de la Comunicación, (13), 2. Recuperado de http://web.upla.cl/revistafaro/n13/art03.htm

CEPAL-UNESCO. (1996). Educación y conocimiento: eje de la transformación productiva con equidad. Lima: Tarea.

Cerón P., Gómez Z. \& Abrego T. (2015). Implementación de B-learning en el Nivel Superior de Educación. Campus Virtuales, 3(2), 8-15. Recuperado de http://www.uajournals.com/ojs/index.php/campusvirtuales/article/view/56

Coll, C. \& Monereo, C. (2008). Psicología de la Educación Virtual. Madrid: Morata.

Collison, G., Elbaum, B., \& Haavind, S. (2000). Facilitating online learning: effective strategies for moderators. USA: Atwood Publishing.

Colmenares, E. (2012). Investigación-acción participativa: una metodología integradora del conocimiento y la acción. Voces y Silencios. Revista Latinoamericana de Educación, 3(1), 102-115. Recuperado de https://revistas.uniandes. edu.co/doi/pdf/10.18175/vys3.1.2012.07

Contreras, R. , Alpiste, F., \& Eguia, J. (2006). Tendencias en la educación: aprendizaje combinado. Theoria, 15(1), 111-117. Recuperado de http://www.redalyc.org/ pdf/299/29915111.pdf

Correa, J., Sierra, M. \& Alzate, G. (2015). Formación de docentes participantes en el programa de educación inclusiva con calidad en Colombia. Revista latinoamericana de educación inclusiva, 9(1), 43-61. Recuperado de https:// dialnet.unirioja.es/servlet/articulo? codigo $=5155476$

Creswell, J. (2003). Research Design. Qualitative, Quantitative, and Mixed Methods Approaches. Second Edition. USA: SAGE Publications.

Díaz, S. (2008). Actos de habla ilocucionarios en dos formas de comunicación mediadas por computador, Un estudio de caso. Bogotá: Tesis de Maestría no publicada, Universidad Pedagògica Nacional, Colombia.

Duncombe, R., \& Heeks, R. (1999). Information, ICTs 
and Small Enterprise: Findings from Botswana. (I. f. Management, Ed.) Development Informatics Working Paper Series. Paper, (7), 1-16.

Escorcia-Oyola, L., \& Jaimes de Triviño, C. (2015). Tendencias de uso de las TIC en el contexto escolar a partir de las experiencias de los docentes. Educación y educadores, 18(1). Recuperado de http://www.redalyc.org/ html/834/83439194008/

Fainholc, B., Nervi, H., Romero, R., \& Halal, C. (2015). La formación del profesorado y el uso pedagógico de las TIC. Revista de Educación a Distancia, (38). Recuperado de http://revistas.um.es/red/article/view/234081

Farroñay, P. \& Ancaya, M. (2016) Gestión administrativa y conocimiento de las TIC en docentes de educación primaria de las instituciones educativas Innova Schools de San Juan de Lurigancho y Ate. Revista Hamut'ay, 3 (1), 31-45. https://doi.org/10.21503/hamu.v3i1.998

Faure, E., Herrera, H., Kaddoura, A., Lopes, H., Petrovski, A., Rahnemma, M., \& Champion, F. (1973). Aprender a Ser. La educación del futuro. Madrid: Alianza-UNESCO.

Fernández, N. (2016) Redes sociales virtuales, ¿̨fortalezas o debilidades? Un análisis psicosocial relacionado con el Cybersexo y la soledad. Revista Hamut'ay, 3 (2), 42-54. https://doi.org/10.21503/hamu.v3i2.1309

Freire T., Gómez Z., \& García V. (2016). Criterios para el diagnóstico de la práctica educativa a distancia en modalidad B Learning. Revista Iberoamericana de Tecnología en Educación y Educación en Tecnología, (17), 67-74. Recuperado de http://teyet-revista.info.unlp.edu.ar/TEyET/article/ view/363

García-Gasco, P. (2003). Software social, conocimiento compartido y toma de decisiones en la orientación académica. II Encuentro Nacional de Orientadores. "Las TIC aplicadas a la Orientación", 465 - 477. Recuperado de http:// www.eldoblao.com/resources/II_Encuentro_completo.pdf

Garrison, D. \& Anderson, T. (2005). El e-learning en el siglo XXI. Barcelona: Octaedro.

Gasca B., Martínez M. \& Tautiva, J. (2016). Comunicación para el cambio social en la emergencia de la alfabetización digital. Revista Nexus Comunicación, 148-167. Recuperado de http://revistaingenieria.univalle.edu.co/index.php/ nexus/article/view/668

González, J. (2008). TIC y la transformación de la práctica educativa en el contexto de las sociedades del conocimiento. Revista de Universidad y Sociedad del Conocimiento (RUSC), 5(2). Recuperado de http://www.uoc.edu/ rusc/5/2/dt/esp/gonzalez.pdf

Graham, C., \& Misanchuk, M. (2004). Computer-Mediated Learning Groups: Benefits and Challenges to Using Groupwork in Online Learning. (T. Roberts, Ed.) Online Collaborative Learning: Theory and Practice, 181-202.

Grande, M., Cañón, R., y Cantón, I. (2016). Tecnologías de la información y la comunicación: Evolución del concepto y características. International Journal of Educational Research and Innovation (IJERI), 6, 218-230. Recuperado de https://rio.upo.es/xmlui/handle/10433/3513

Gutiérrez, D. (2016). El desempeño docente y el uso de recursos informáticos en la Institución Educativa No 7050 “Nicanor Rivera Cáceres”. Hamut'ay, 3(1), 46-58. https:// doi.org/10.21503/hamu.v3i1.999

Gutiérrez-Santiuste, E., \& Gallego-Arrufat, M. (2017). Presencia social en un ambiente colaborativo virtual de aprendizaje: Análisis de una comunidad orientada a la indagación. Revista Mexicana de Investigación Educativa, 22(75). Recuperado de http://www.comie.org.mx/documentos/rmie/ v22/n075/pdf/75007.pdf

Henao, O. (2001). Identificación de ideas importantes y detalles en textos hipermediales e impresos. Lectura y Vida. Revista Latinoamericana de lectura, 22(3), 6-15. Recuperado de http://www.lecturayvida.fahce.unlp.edu.ar/numeros/ a22n3/22_03_Henao.pdf

Jiménez, N. (2009). Los medios de comunicación frente a la revolución de la información. El Cid Editor. Santafé, Argentina: El Cid.

KENTA. (2009). Promoción del trabajo colaborativo a través de la integración de objetos de aprendizaje en ambientes virtuales. Bogotá: Centro de Investigaciones Universidad Pedagógica Nacional - CIUP.

Larrosa, J. (2003). Algunas notas sobre experiencia y sus lenguajes. Serie Encuentros y Seminarios. Conferencia Universidad de Barcelona. Recuperado de http://www.me.gov. ar/curriform/publica/oei_20031128/ponencia_larrosa.pdf

Llorente, M. (2006). El tutor en E-learning: aspectos a tener en cuenta. Edutec. Revista Electrónica de Tecnología Educativa, (20). Recuperado de http://edutec.rediris.es/Revelec2/ revelec20/llorente.htm

Luján, R. (2016). Enseñanza de las TIC para el desarrollo de competencias tecnológicas en docentes. Hamut'ay, 3(1), 19-30. https://doi.org/10.21503/hamu.v3i1.997

Montenegro, D. (2016) Interacción comunicativa con Blackboard Collaborate y el rendimiento académico en estudiantes de educación a distancia. Revista Hamut'ay, 3 (2), 68-82. https://doi.org/10.21503/hamu.v3i2.1322

Najmanovich, D. (2009). Educar y aprender: Escenarios y paradigmas, en Dossier Pensar la cultura: saberes, imaginarios y sujetos de la contemporaneidad. Revista Propuesta Educativa, 32, 11-22. Recuperado de http://www.redalyc. org/pdf/4030/403041704008.pdf

Olaizola, E. \& Peralta, M. (2017). Estrategias y desafíos del rol tutorial en una propuesta de posgrado a distancia internacional. En: IV Jornadas de TIC e Innovación en el Aula (La Plata, 2017). Recuperado de http://sedici.unlp.edu.ar/ bitstream/handle/10915/66323/Documento_completo. pdf-PDFA.pdf?sequence $=1$

Orellana L. \& Sánchez G. (2006). Técnicas de recolección de datos en entornos virtuales más usadas en la investigación cualitativa. Revista de Investigación Educativa, 2006, 24(1), 205-222. Recuperado de http://revistas.um.es/rie/article/ view/97661/93701 
Rodríguez, G. \& Valldeoriola R. (2001). Metodología de la investigación. Cataluña: Universitat Oberta de Cataluña. Recuperado de http://myuvmcollege.com/uploads/lectura2011-09/Metodolog\%C3\%ADa\%20de\%20investigaci\%C3\%B3n-2064.pdf

Rodríguez, S. \& López, R. (2017). Estrategias de enseñanza en los entornos mediados: resultados de la experiencia de la performance virtual educativa. Revista de Educación a Distancia, (55). http://dx.doi.org/10.6018/red/55/10

Rodríguez, J. (2007). Narrativa, juego y conocimiento.La iniciativa digital en acción en Golpe de Gracia. Cuadernos de Literatura, 12(23), 106 - 117. Recuperado de http:// www.erevistas.csic.es/ficha_articulo.php?url=oai:ojs.www. javeriana.edu.co:article/6558\&oai_iden=oai_rev

Ruíz, M. \& Aguirre, G. (2013). Quehacer docente, TIC y educación virtual o a distancia. Apertura. Revista de innovación educativa, 5(2), 108-123. http://www.redalyc.org/ pdf/688/68830444010.pdf

Sánchez, C. (2015). B-learning como estrategia para el desarrollo de competencias. El caso de una universidad privada. Revista Iberoamericana de Educación, 67(1), 85-100. Recuperado de https://rieoei.org/RIE/article/view/265

Scolari, C. (2008). Hipermediaciones: elementos para una teoría de la comunicación digital interactiva. Barcelona: Gedisa.

Sein-Echaluce L., Fidalgo B. \& García P. (2015). Metodología de enseńanza inversa apoyada en b-learning y gestión del conocimiento. Recuperado de https://repositorio.grial. eu/handle/grial/480

Silva, J. (2007). Las Interacciones en un Entorno Virtual de Aprendizaje para la Formación Continua de Docentes de Enseñanza Básica (tesis doctoral). Universidad de Barcelona, Barcelona, España.

Silva, J. (2017). Un modelo pedagógico virtual centrado en las E-actividades. Revista de Educación a Distancia, (53). Recuperado de http://revistas.um.es/red/article/view/290021

Simons, M., \& Masschelein, J. (2013). Se nos hace creer que se trata de nuestra libertad: notas sobre la ironía del dispositivo de aprendizaje. Revista Pedagogía y Saberes, 38, 93-102. https://doi.org/10.17227/01212494.38pys93.102

Vega, V. (2017). El trabajo colaborativo a través de la historia de las TIC. Revista Q, 4(7). Recuperado de https:// revistas.upb.edu.co/index.php/revista_Q/article/view/7814

Viñals, A. \& Cuenca, J. (2016). El rol del docente en la era digital. Revista Interuniversitaria de Formación del Profesorado, 30(2), 103-114. Recuperado de http://www.redalyc. org/pdf/274/27447325008.pdf

Vinueza V. \& Simbaña G. (2017). Impacto de las TIC en la Educación Superior en el Ecuador. Revista Publicando, 4(11), 355-368. Recuperado de https://rmlconsultores. com/revista/index.php/crv/article/view/530

WCEFA. (1990). Educación para todos y marco de acción para satisfacer las necesidades básicas del aprendizaje. Jomtiem. in/poll/570/
Zoll, C., Enz, S., Schaub, H., Aylett, R., \& Paiva, A. (2006). Fighting bullying with the help of autonomous agents in a virtual school environment. Paper presented at the 7 th International Conference on Cognitive Modelling. 